\title{
One-pot synthesis of sulfur heterocycles from simple organic substrates
}

\author{
Oleg A. Rakitin \\ N. D. Zelinsky Institute of Organic Chemistry, Russian Academy of Sciences, 47, Leninsky \\ prosp., 119991 Moscow, Russian Federation \\ E-mail: orakitin@ioc.ac.ru
}

\begin{abstract}
Sulfur-nitrogen heterocycles are important compounds due to their unusual physical properties and significant and versatile biological activity. A novel strategy for their preparation from readily available organic substrates with the employment of sulfur monochloride is proposed. A synthesis of wide range of heterocyclic systems including 1,2-dithioles, 1,2,3,4,5-pentathiepins, 1,2,3-dithiazoles, 1,4-thiazines, 1,2,3,4,5,6,7-heptathiocanes and others, in a cascade transformations has been developed.
\end{abstract}

Keywords: Sulfur monochloride, heterocycles, one-pot synthesis, 1,2-dithioles, 1,2,3,4,5pentathiepins, tertiary amines

\section{Table of Contents}

1. Introduction

2. Reaction of Sulfur Monochloride with Tertiary Amines. Synthesis of 1,2-Dithioles

3. Synthesis of Fused 1,2,3,4,5-Pentathiepins

4. Synthesis of Other Sulfur Containing Heterocycles

5. Conclusions

Acknowledgments

References

\section{Introduction}

Sulfur-nitrogen heterocyclic compounds have retained the interest of organic and inorganic researchers along decades of historical development of chemistry. The presence of heteroatoms results in significant changes in the cyclic molecular structure due to the availability of unshared 
pairs of electrons and the difference in electronegativity between heteroatoms and carbon. As a result there is an unique feature of these heterocycles which are considered as promising materials in different areas of pharmaceutical and agrochemical research and also more recently as compounds with interesting physical properties especially for magnetism and conductivity. However the preparation of these compounds by conventional methods has usually required many synthetic steps and expensive starting materials.

More than fifteen years ago a novel strategy for the synthesis of complex polysulfurnitrogen heterocycles from simple (sometimes commercially available) organic substrates and sulfur monochloride $\left(\mathrm{S}_{2} \mathrm{Cl}_{2}\right)$ has been proposed. This idea has been based on the complex and versatile reactivity of sulfur monochloride. ${ }^{1} \mathrm{~S}_{2} \mathrm{Cl}_{2}$ is currently used for perchlorination of aromatic systems. ${ }^{2,3}$ Also sulfur monochloride can be considered as a reactive electrophile $e^{4,5}$ and oxidant (see examples in review [1]). But the sulfurating ability of sulfur monochloride is more important. All these abilities of sulfur monochloride can take place simultaneously and this, from one side, diminishes its value as selective sulfurating agent, and, from the other side, leads to extensive cascade transformations with the formation of complex heterocyclic compounds.

Sulfur monochloride is highly reactive towards organic substrates containing many functional groups. We have found that chemical substances containing activated $\mathrm{C}-\mathrm{H}$ bonds, nitrile and imino groups can be involved in the reaction with $\mathrm{S}_{2} \mathrm{Cl}_{2}$. This review is devoted to these previously unknown transformation of simple organic substances into complex polysulfurnitrogen containing heterocycles.

\section{Reaction of Sulfur Monochloride with Tertiary Amines. Synthesis of 1,2- Dithioles}

One of the most exciting reactions investigated by us is the reaction with trialkylamines. In fact, reaction with ammonia, primary and secondary amines has been investigated before and did not afford heterocyclic compounds which we are interested in. For example, reaction of sulfur monochloride with ammonia in a form of its salt (ammonium chloride) leads to tetrasulfur tetranitride. ${ }^{6}$ Primary and secondary amines reacted with sulfur monochloride easily to give corresponding disulfides (Scheme 1).

$$
\begin{gathered}
\mathrm{S}_{2} \mathrm{Cl}_{2}+\mathrm{NH}_{4} \mathrm{Cl} \longrightarrow \mathrm{S}_{4} \mathrm{~N}_{4} \\
\mathrm{R}_{2} \mathrm{NH}+\mathrm{S}_{2} \mathrm{Cl}_{2} \longrightarrow \mathrm{R}_{2} \mathrm{~N}-\mathrm{S}-\mathrm{S}-\mathrm{NR}_{2} \\
\mathrm{R}=\mathrm{H}, \mathrm{Alk}, \mathrm{Bn}, \mathrm{Ph}, \mathrm{C}(\mathrm{O}) \mathrm{R}^{1} \text { etc } \quad \text { More than } 35 \text { reactions }
\end{gathered}
$$

Scheme 1. Reaction of $\mathrm{S}_{2} \mathrm{Cl}_{2}$ with ammonium chloride, primary and secondary amines. 
During our work we have unexpectedly found that sulfur monochloride reacted with practically all trialkylamines investigated. But also one of the most interesting finding is that at low temperature for some tertiary amines and at room temperature for 1,4-diazabicyclooctane (DABCO) sulfur monochloride formed reactive complexes. ${ }^{7}$ Thus, a mixture of equimolar amounts of $\mathrm{S}_{2} \mathrm{Cl}_{2}$ and $\mathrm{DABCO}$ in chloroform, stored for $1 \mathrm{~h}$ at room temperature before use, gave different products to those formed when the heterocycle, $\mathrm{S}_{2} \mathrm{Cl}_{2}$ and DABCO were all mixed together at the beginning. We assume that complexes are formed between $\mathrm{S}_{2} \mathrm{Cl}_{2}$ and $\mathrm{DABCO}$ and we studied their solutions in chloroform by infrared spectroscopy. The S-S absorption band is shifted in the $1: 1$ mixture from $540 \mathrm{~cm}^{-1}$ to $580 \mathrm{~cm}^{-1}$, and the $\mathrm{S}-\mathrm{Cl}$ absorption bands of $\mathrm{S}_{2} \mathrm{Cl}_{2}$ (v 436 and $452 \mathrm{~cm}^{-1}$ ) are present in the 1:1 mixture of $\mathrm{S}_{2} \mathrm{Cl}_{2}$ and $\mathrm{DABCO}$, but disappear when a second mole of DABCO is added. We assume that the 1:1 mixture contains complex 1 predominantly, and the 1:2 mixture - complex 2 predominantly (Scheme 2).

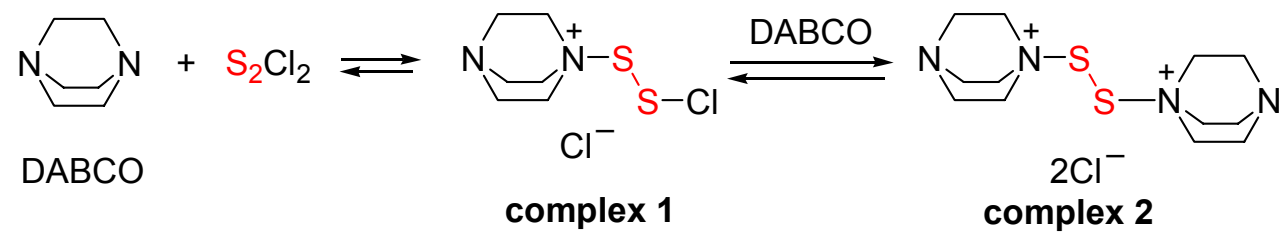

Scheme 2. Complexes of $\mathrm{S}_{2} \mathrm{Cl}_{2}$ and $\mathrm{DABCO}$.

More than ten years ago we discovered that $N$-ethyldiisopropylamine (so called Hünig's base), having been initially used as an "inert" base, reacted with sulfur monochloride and DABCO by a single step reaction to give an unexpected and novel multisulfur-nitrogen system tricyclic bis(dithiolo)thiazine dithione $1{ }^{8}$ In this one-pot conversion of Hünig's base tricycle has been obtained in a one-pot reaction, by mixing two simple commercially available compounds in high yield under mild conditions, to produce a new heterocyclic system by an entirely new method; besides 14 isopropyl $\mathrm{C}-\mathrm{H}$ bonds were replaced by $10 \mathrm{C}-\mathrm{S}$ and two $\mathrm{C}-\mathrm{C}$ double bonds, while the ethyl group remained intact (Equation 1).
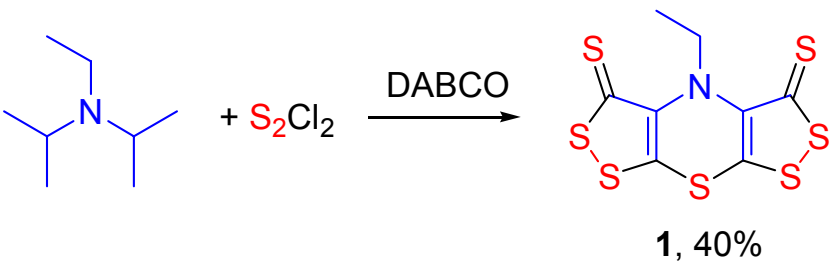

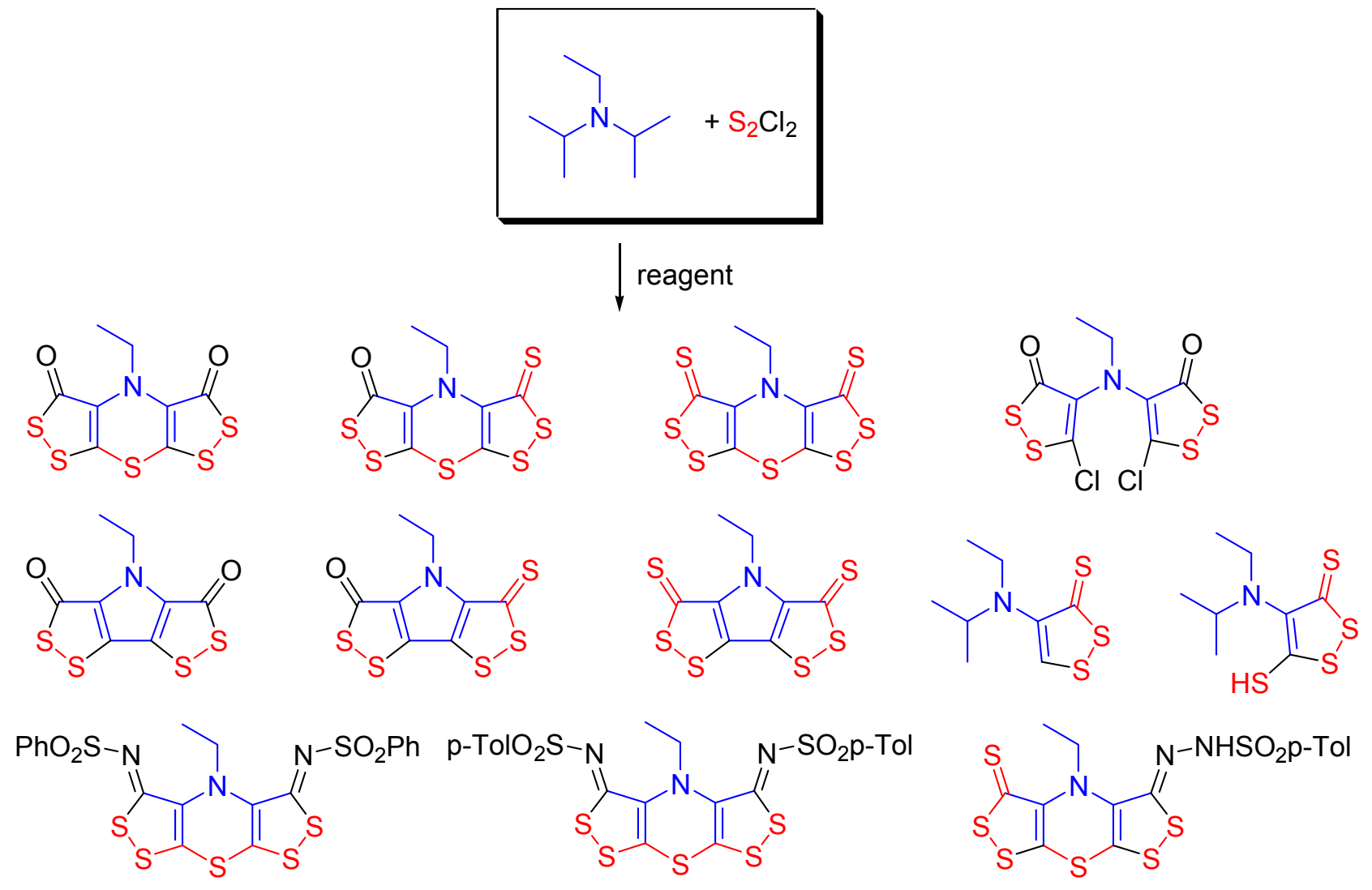<smiles>CCN(c1ssc(=S)c1Nc1ccccc1)c1c(Nc2ccccc2)ssc1=S</smiles>
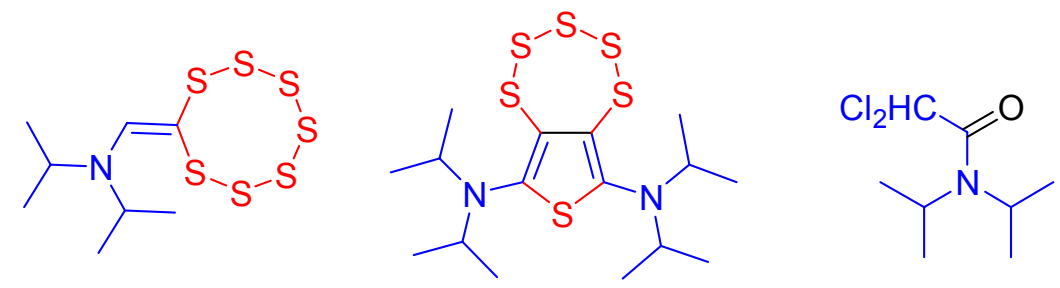

Scheme 3. Compounds obtained from the reaction of $\mathrm{S}_{2} \mathrm{Cl}_{2}$ and $N$-ethyldiisopropylamine.

This was a remarkable reaction between two reagents; it turned out that from these two compounds: Hünig's base and sulfur monochloride following addition of various substances 16 compounds can be isolated all in one-pot conversions; some of them selectively and in high yields (Scheme 3). ${ }^{9-13}$

A good combination of reagents and reaction sequences permits the selective preparation of most of these compounds and can imply up to fifteen different steps all working sequentially in a one-pot reaction. Let's consider the plausible mechanism for the formation of these compounds (Scheme 4). ${ }^{9}$ The first step is an oxidation of the amine to an immonium salt $\mathbf{2}$ by a combination of sulfur monochloride and DABCO. The immonium salt can subsequently be converted into a reactive enamine 3 trapped by another molecule of $\mathrm{S}_{2} \mathrm{Cl}_{2}$ with cyclization to a dithiole ring 4 . Then the chlorination and dehydrochlorination of the dithiole ring affords a stable salt $\mathbf{5}$. Repeating the reaction on the other isopropyl group successfully converts the starting material into the crucial 
intermediate disalt $\mathbf{6}$. The disalt $\mathbf{6}$ is very sensitive to the reaction conditions. In the presence of $\mathrm{S}_{2} \mathrm{Cl}_{2}$ and DABCO in equimolecular amounts, a new cyclization step, followed by extrusion of sulfur, gives rise to a new disalt 7, but in the presence of excess sulfur monochloride chlorination is favored affording chlorinated disalt $\mathbf{8}$. These reactive 1,2-dithiolium salts 5-8 undergo reaction with nucleophiles that already presented in the reaction environment or added in the last period of the reaction. In general, the multicomponent mixture works with a high efficiency, permitting the selective preparation of the most final products.
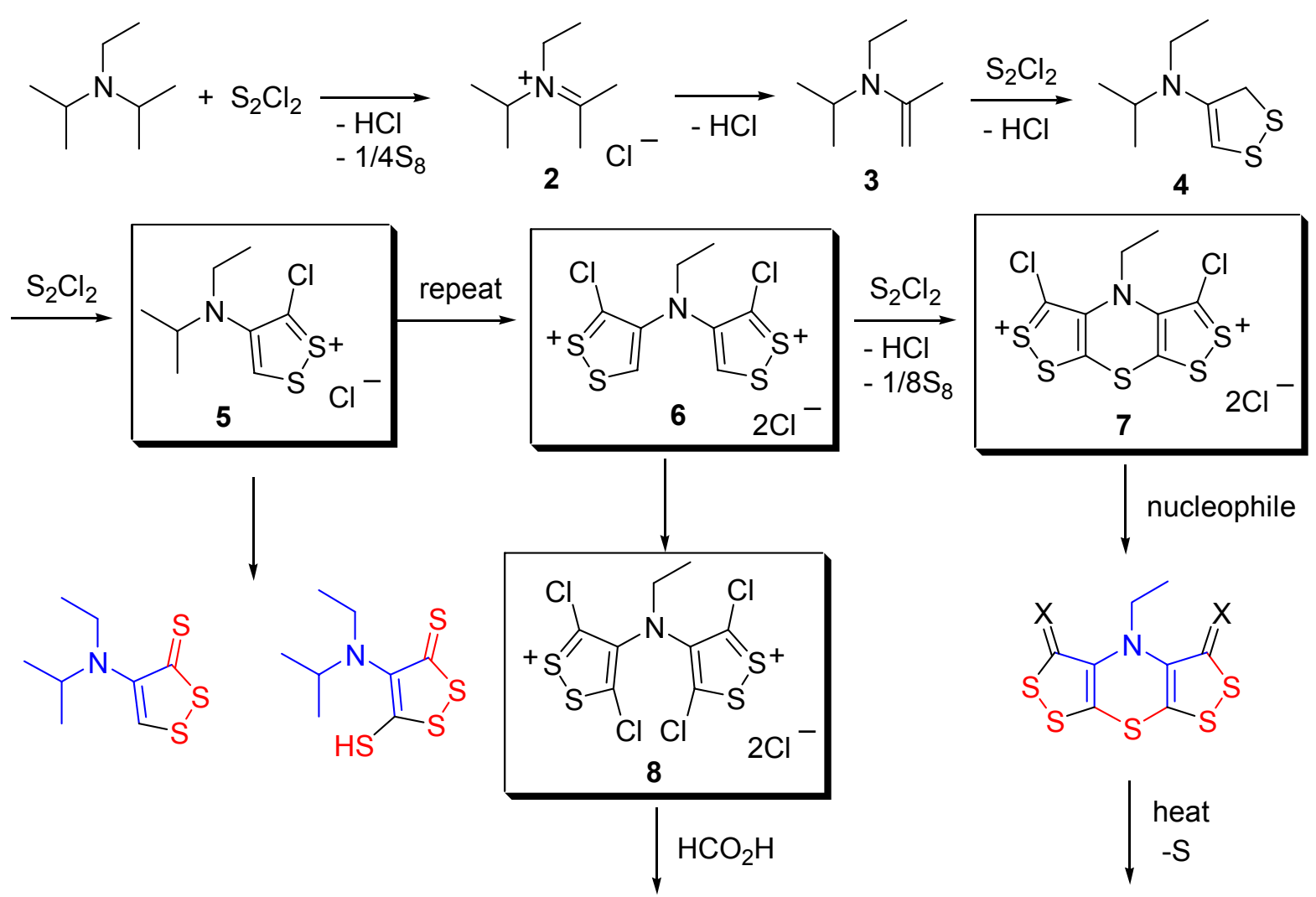

$\mathrm{X}=\mathrm{O}, \mathrm{S}, \mathrm{N}-\mathrm{SO}_{2} \mathrm{Ar}$
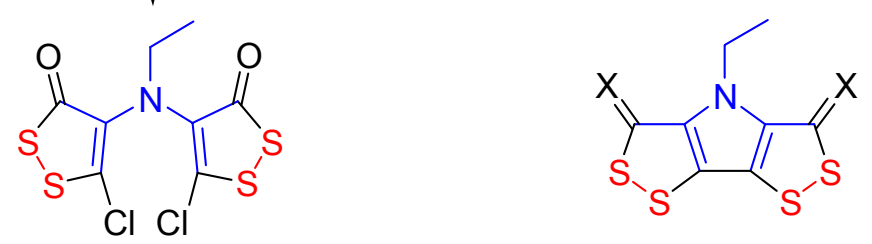

Scheme 4. Plausible mechanism for the reaction between $\mathrm{S}_{2} \mathrm{Cl}_{2}$ and $N$-ethyldiisopropylamine.

All tricyclic $N$-substituted bisdithiolothiazines such as $\mathbf{1}$ adopt bent conformation. But there is one exception to this molecular habit. Thus, the parent ring, having a hydrogen on the nitrogen, is a planar compound in the solid state. This compound 9 is obtained by debenzylation of the $N$-benzyl substituted bis[1,2]dithiolo[1,4]thiazine derivative (Equation 2). ${ }^{14}$ The unsubstituted ring system has shown complete inhibitory activity against some tumor strains at low concentration $\left(10^{-4} \mathrm{M}\right)$ in the preliminary in vitro screenings. 
<smiles>O=c1ssc2c1N(Cc1ccccc1)c1c(ssc1=O)S2</smiles><smiles>O=c1ssc2c1CNc1c-2ssc1=O</smiles>

The $N$-(2-chloroethyl)diisopropylamine 10 constitutes a very special case. Its reaction with sulfur monochloride, whether or not in the presence of formic acid, gives the tricyclic bis[1,2]dithiolo[1,4]thiazine derivatives. The course of the reaction is completely changed by addition of phosphorus pentasulfide at the last stage of the reaction. In this case, the chlorine atom is replaced by sulfur both in the lateral chain or in the intermediate salt, thus giving a new $[1,2]$ ditiolo[1,4]thiazine ring system. ${ }^{15}$ This new compound $\mathbf{1 1}$ has shown a notable antitumor activity against breast cancer cells at low concentration $\left(10^{-4} \mathrm{M}\right)$.

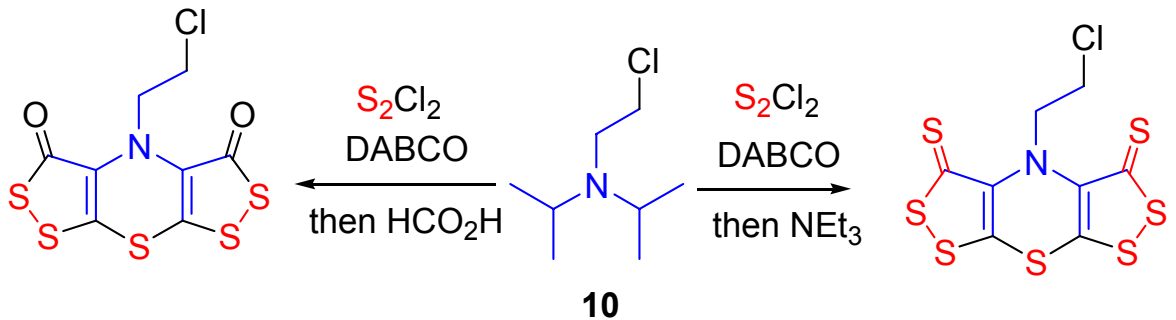

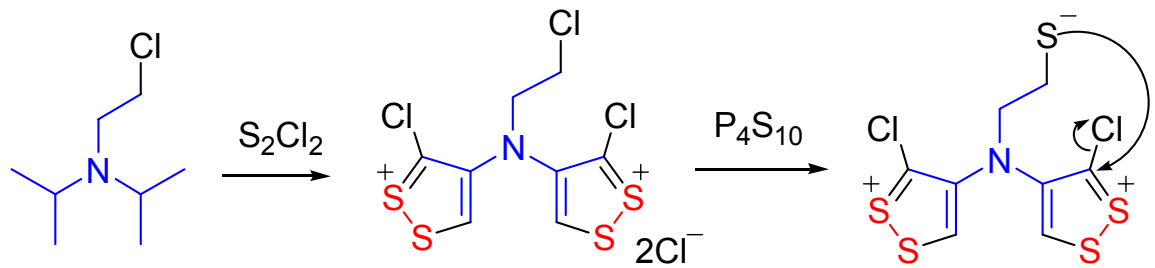

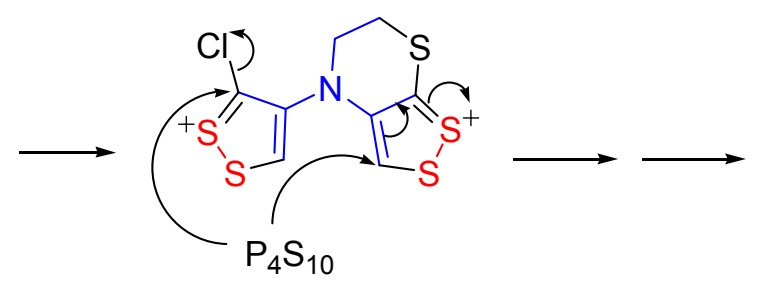<smiles>S=c1sscc1N1CCSc2ssc(=S)c21</smiles>

Scheme 5. Reaction of $N$-chloroethyldiisopropylamine 10 with $\mathrm{S}_{2} \mathrm{Cl}_{2}$.

The suggested mechanism for all these transformations involves as a key step transformation of isopropyl group into 3-chlorodithiolium salt 5. Going further with the investigation in this area, we demonstrated that the reaction can be stopped at the step of formation of monocyclic 1,2-dithioles. ${ }^{16}$ The main condition for the successful synthesis of monodithioles is low temperature $\left(-15^{\circ} \mathrm{C}\right)$. In agreement with the proposed mechanism the combination of an excess 
of sulfur monochloride over tertiary amine and DABCO is expected to yield dichlorodithiolium salt 12 which in the presence of a oxygen nucleophile (formic acid) gives 5-chloro-dithiol-3-one 13 (Scheme 6). Our synthesis of 1,2-dithiole-3-ones under unusually mild conditions is exclusive among known relevant methods and provides new wide possibilities for the study of this promising chemical class.

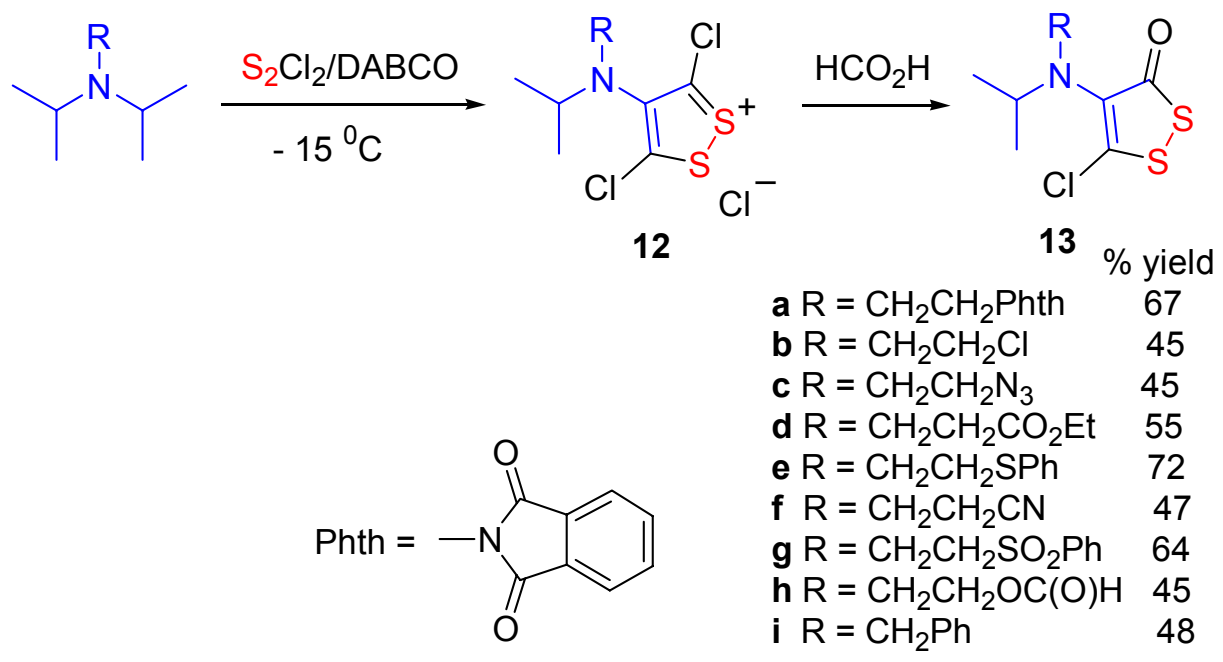

Scheme 6. Synthesis of monocyclic 5-chloro-1,2-dithiol-3-ones 13.

We decided to use 5-chloro-1,2-dithioles $\mathbf{1 3}$, currently readily available, in further reactions with sulfur monochloride. It was quite unusual idea because these compounds were prepared with $\mathrm{S}_{2} \mathrm{Cl}_{2}$. The reaction of dithiolone 13a with $\mathrm{S}_{2} \mathrm{Cl}_{2}$ and $\mathrm{DABCO}$ was investigated in detail; the nature of the solvent used appeared to be crucial for the successful reaction course. If the reaction was carried out in an inert solvents such as chloroform, dichloromethane or 1,2dichloroethane, starting dithiolone 13a was isolated in practically quantitative yield. The attempts to employ other solvents, recently used in reactions with sulfur monochloride (THF or $\mathrm{DMF}$ ), were failing; neither a reaction (in the first case) nor decomposition (in the second case) is occurred. Treatment of $\mathrm{S}_{2} \mathrm{Cl}_{2}$ and DABCO in acetonitrile - a solvent which is rarely used in $\mathrm{S}_{2} \mathrm{Cl}_{2}$ reactions - gave bisdithiolothiazine ketothione 14a after treatment with triethylamine as a base. We then extended this reaction to other $N$-substituted 4-isopropylamino-5-chloro-1,2dithiole-3-ones 13. ${ }^{17}$ Bisdithioloketothiones 14 were obtained selectively in all reactions in moderate to high yields (Scheme 7). 


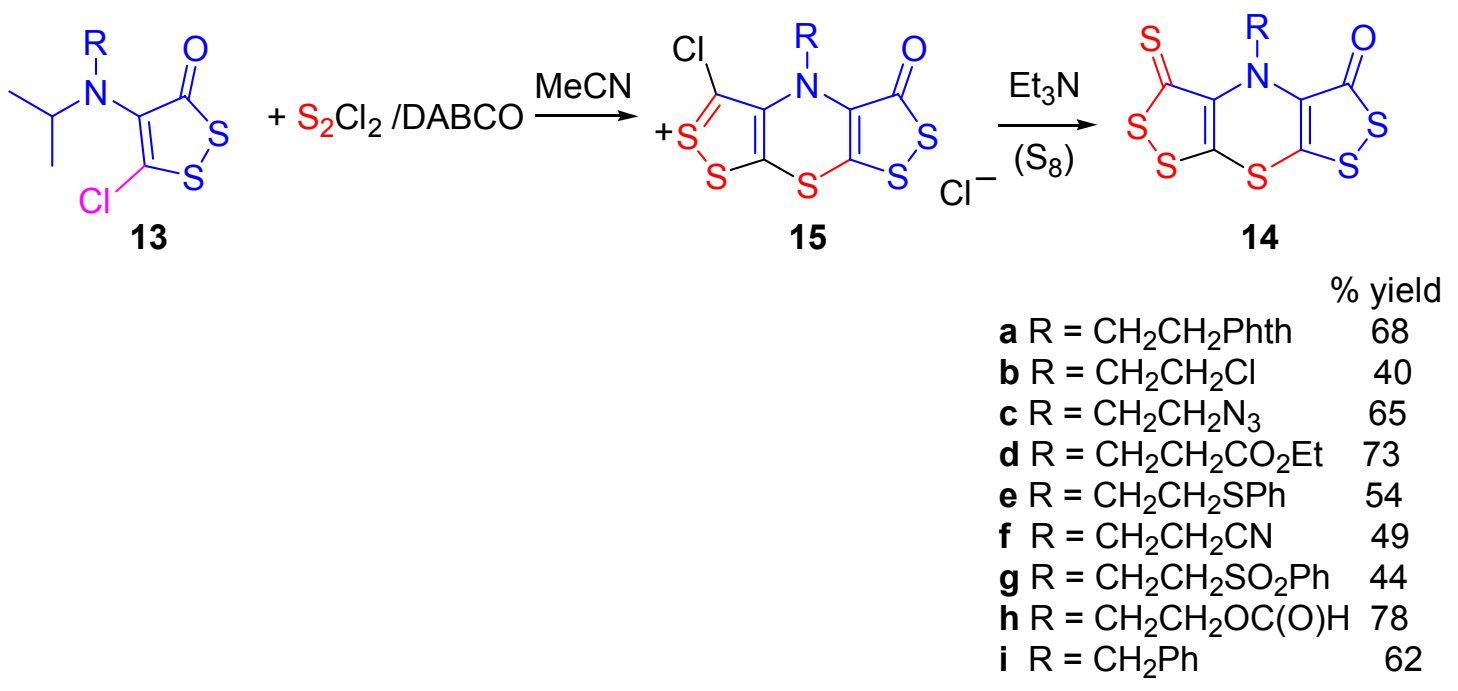

Scheme 7. Synthesis of bisdithiolothiazine ketothiones 14.

We believe this to be the first case where a chloro substituent is replaced by sulfur under the electrophilic action of sulfur monochloride and a base. Presumably the key intermediate in the reaction is 3-chlorodithiolium salt $\mathbf{1 5}$ which gave thiones $\mathbf{1 4}$ with sulfur nucleophiles generated from a base (e.g. triethylamine) and sulfur formed in the ongoing reaction. Assumingly, oxygen nucleophile (most frequently used formic acid) could give diketone 16. We checked this possibility by replacing $\mathrm{Et}_{3} \mathrm{~N}$ by formic acid at the last step. In fact, diketones $\mathbf{1 6}$ were prepared selectively with moderate yields (Scheme 8).

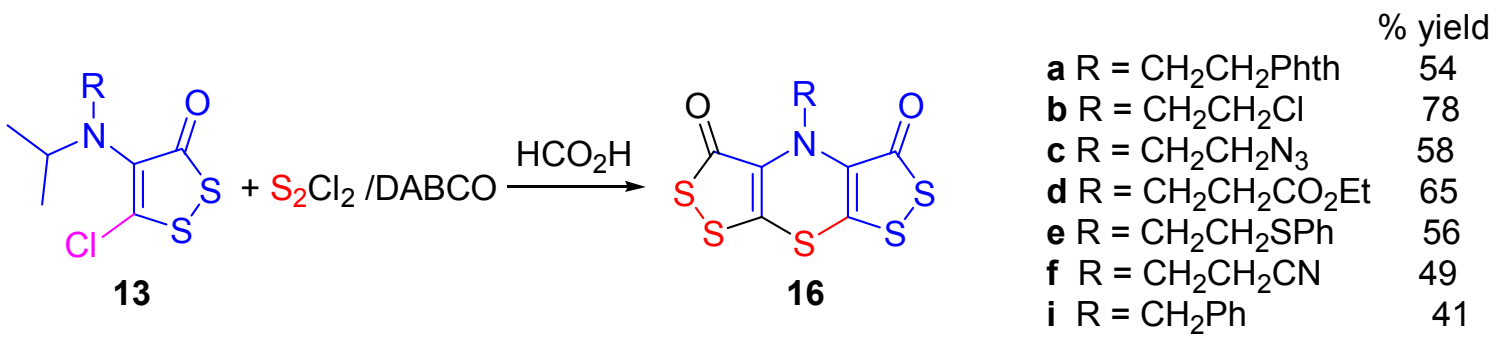

Scheme 8. Synthesis of bisdithiolothiazine diketones 16.

In an attempt to extend the $\mathrm{S}_{2} \mathrm{Cl}_{2}$ sulfurating ability in reactions with 5-chloro-1,2-dithioles, we investigated $N, N$-bis(5-chloro-3-oxo[1,2]dithiol-4-yl)amines 17 in a reaction with sulfur monochloride and a base. $\mathrm{DABCO}$ was found to be inert in this reaction which was not surprising bearing in mind that bicyclic compounds $\mathbf{1 7}$ were formed in the presence of DABCO. Treatment with $\mathrm{S}_{2} \mathrm{Cl}_{2}$ and triethylamine in chloroform for $3 \mathrm{~d}$ at room temperature followed by heating under reflux for $3 \mathrm{~h}$ gave bisdithiolothiazines 16 in high yields. The best results were obtained where $\mathrm{Et}_{3} \mathrm{~N}$ was in a 3 -fold excess with respect to $\mathrm{S}_{2} \mathrm{Cl}_{2}$. 
<smiles>[R]N(c1c(Cl)ssc1=O)c1c(Cl)s[se+]([SeH])c1=O</smiles>

17<smiles></smiles>

16

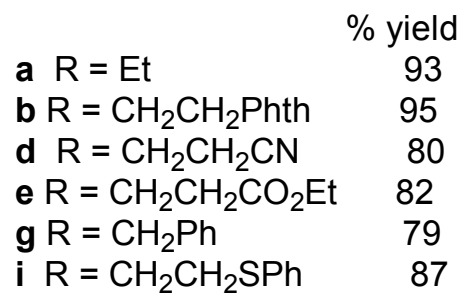

Scheme 8. Reaction of bis(dithiolyl)amines 17 with $\mathrm{S}_{2} \mathrm{Cl}_{2}$.

The novelty of these transformations is in replacing chlorines by sulfur in the reaction with electrophilic sulfur monochloride and its mixtures with tertiary amines. The key steps may be explained by the addition of sulfur monochloride into $\mathrm{C}-\mathrm{H}$ or $\mathrm{C}-\mathrm{Cl}$ bonds with further extrusion of $\mathrm{SCl}_{2}$. These feasible experimental procedures may serve as an efficient basis for new syntheses of sulfur compounds from readily available chloro derivatives.

To continue investigation of the synthetic utility of $N$-ethyldiisopropylamine, we increased the reaction temperature from $-15{ }^{\circ} \mathrm{C}$ to $0{ }^{\circ} \mathrm{C}$. Unexpectedly, these conditions led to an entirely different reaction in which the isopropyl groups are unchanged and the ethyl group is transformed into a dichloroacetyl group 18, and it is a new transformation (Scheme 9). ${ }^{13}$ To see if other ethyl or substituted ethyl groups can be involved in this transformation we investigated the reaction of some tertiary amines with sulfur monochloride and DABCO at $0{ }^{\circ} \mathrm{C} . \mathrm{N}-(2-$ chloroethyl)diisopropylamine gave the same as in the case of Hünig's base dichloroacetyl compound 18. Similar products were obtained from diethylisopropyl- and triethylamines (Scheme 9).

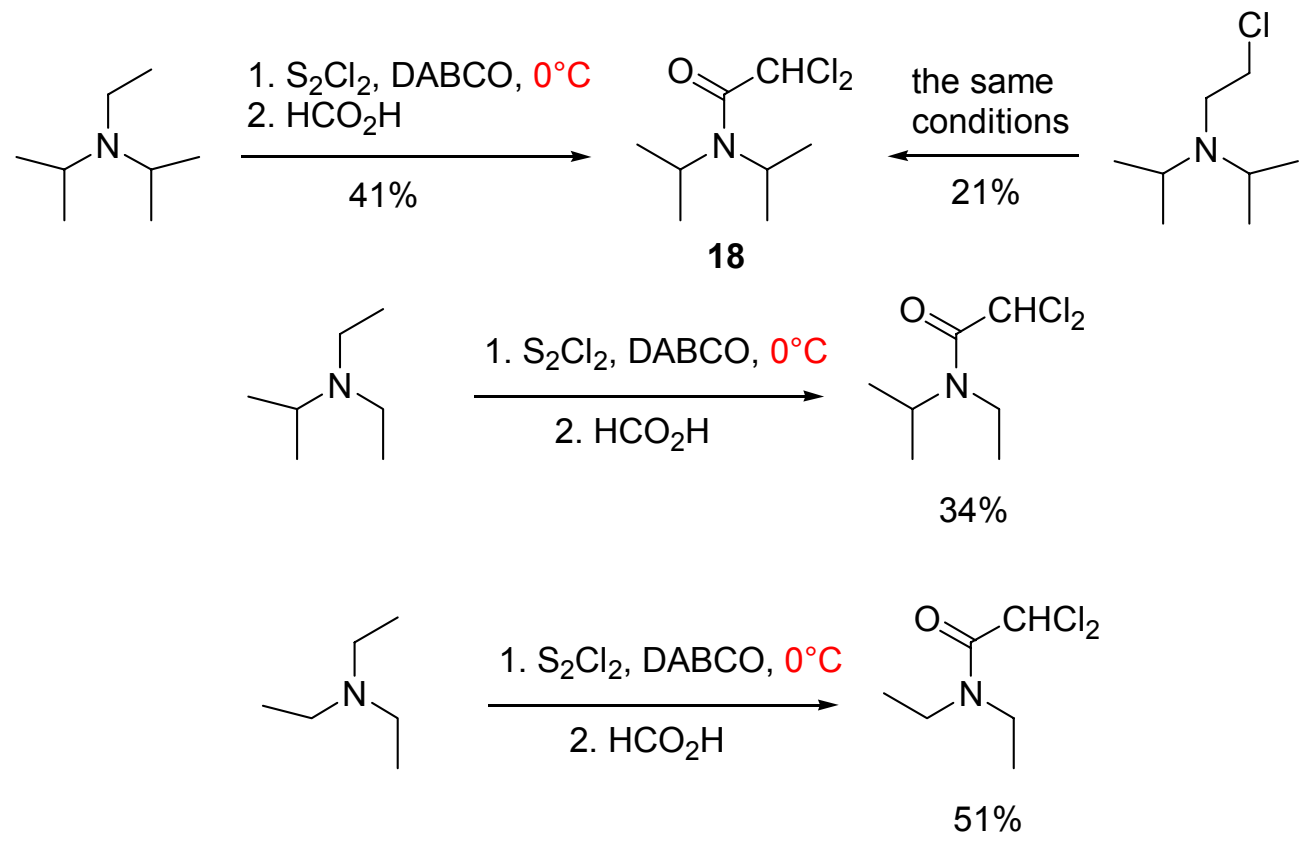

Scheme 9. Synthesis of dichloroacetyl derivatives. 
It may be hard to believe how further changing of the reaction conditions can change the reaction path. But, if we used in the reaction with triethylamine, not a simple mixture of sulfur monochloride and DABCO, but their complex 1, we isolated two entirely new and unexpected substances - thienopentathiepin 19a and heptathiocane 20a, different from all those produced when reagents were not premixed and equilibrated (Scheme 10). ${ }^{18}$ The first compound is the product of an unprecedented conversion of triethylamine into pentathiepin, in which a thiophene ring is created from two $N$-ethyl groups with the formation of a carbon-carbon bond between unactivated methyl groups. We have found that the two products were not interconverted under reaction conditions and were presumably formed in simultaneous, competing reactions. The structure heptathiocane 20a has been confirmed by X-ray analysis.

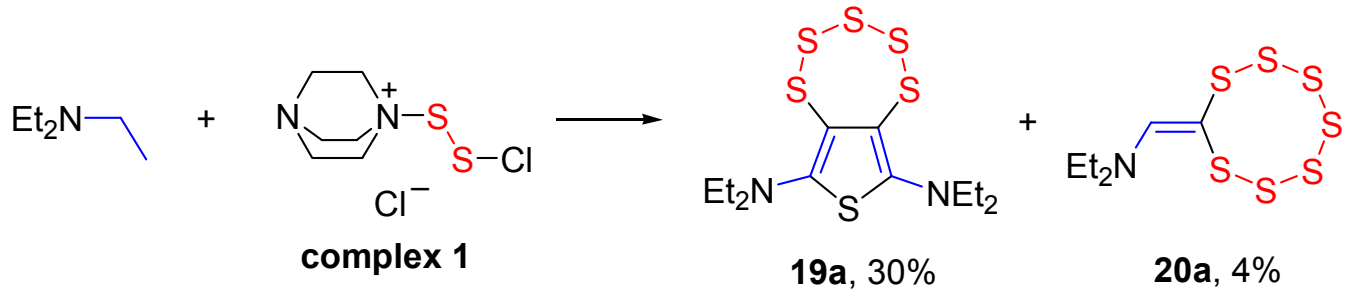

Scheme 10. Reaction of triethylamine with complex 1.

Given that these are reactions of the ethyl group, $\mathrm{Et}_{3} \mathrm{~N}$ should be a favored substrate; the same reactions were observed with other tertiary $N$-ethylamines but in lower yields. Diethyl $n$ propylamine, ethyldiisopropylamine, benzyldiethylamine, dibenzylethylamine, and $\mathrm{N}$ ethylpiperidine all gave thienopentathiepins $19(1-28 \%)$ and corresponding heptathiocanes 20 $(3-10 \%)$ (Scheme 11). The structure of the bispiperidinothienopentathiepin was confirmed by $\mathrm{X}$-ray diffraction. The pentathiepin ring have the expected chair-type conformation. Whilst the yields of these products were mostly low, they are readily prepared in one pot from cheap starting materials. The conversion of $\mathrm{Et}_{3} \mathrm{~N}$ into pentathiepin and heptathiocane both involve an oxidative reaction of one ethyl group together with several other transformations. The two products isolated are probably the most stable members of polysulfur rings containing two and one $s p^{2}$ carbons respectively.

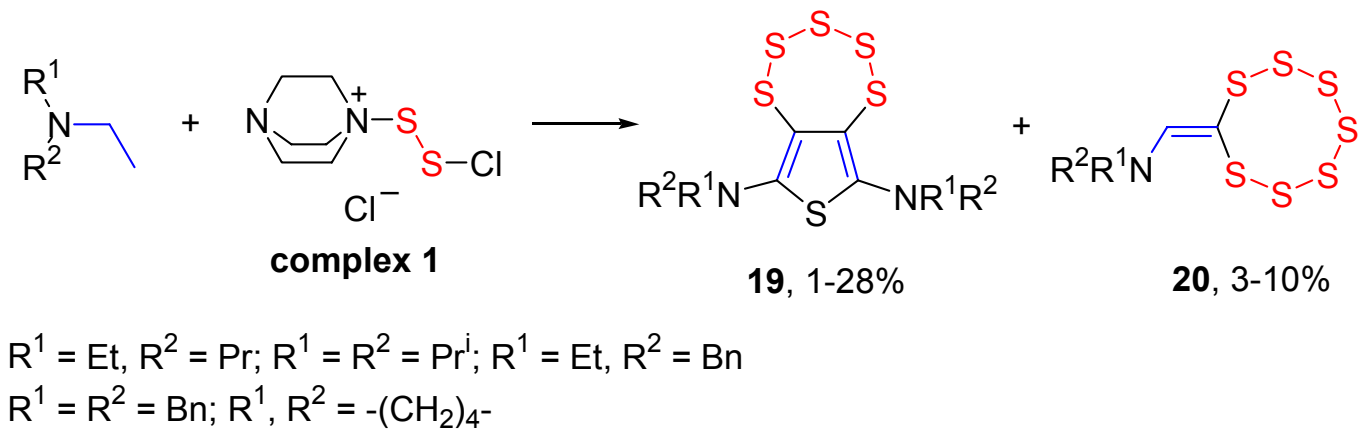

Scheme 11. Synthesis of thienopentathiepins 17 and heptathiocanes 18. 


\section{Synthesis of Fused 1,2,3,4,5-Pentathiepins}

We found that the treatment of simple nucleophilic heterocycles like pyrroles and thiophene, and their tetrahydro derivatives with sulfur monochloride and DABCO provides a simple one-pot synthesis of mono- and bispentathiepins. Actually this new transformation was discovered by pure accident when we investigated the reaction of $N$-isopropylpyrrole with sulfur monochloride trying to obtain $N$-1,2-dithiole-3-thione 21 (Scheme 12). But it was found that the pyrrole ring is more reactive than the isopropyl group. This unusual reaction could thus provide an attractive route to fused pentathiepins and we have studied this reaction systematically to find the best reaction conditions and to explore its scope.

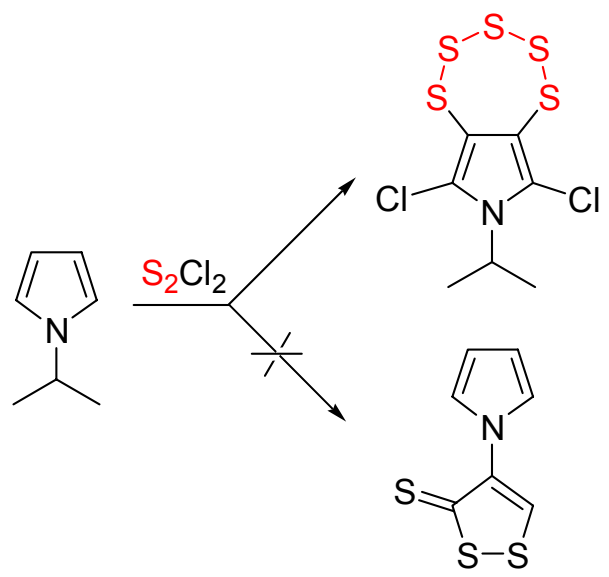

21

Scheme 12. Discovery of fused 1,2,3,4,5-pentathiepins synthesis.

The reaction of $N$-methylpyrrole with sulfur monochloride and DABCO gave dichloropyrrolopentathiepin 22a in best conditions in 50\% yield. ${ }^{19}$ In the formation of 22a from $\mathrm{N}$-methylpyrrole, a pentathiepin ring has been fused to the pyrrole ring and both pyrrole $\alpha$ positions have been chlorinated. It is not surprising that 2,5-dichloro- and 2- chloropyrroles gave in the same way pyrrolopentathiepin 22a even in higher yields (Scheme 13).

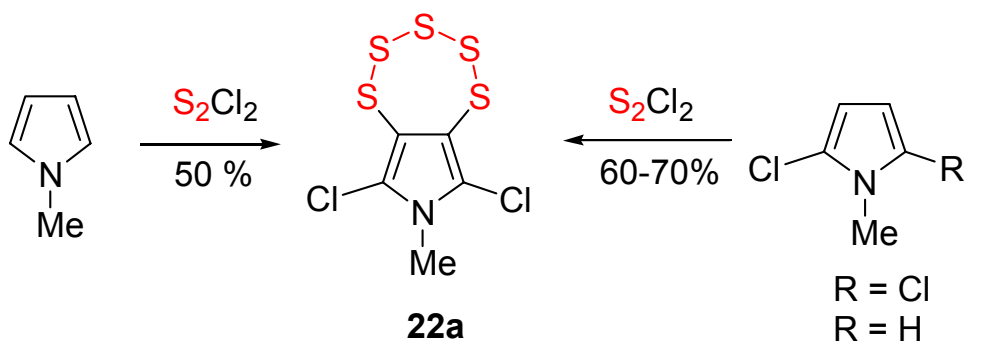

Scheme 13. Synthesis of dichloropyrrolopentathiepin 22a. 
Since $\mathrm{S}_{2} \mathrm{Cl}_{2}$ could also, in principle, oxidize the pyrrolidine to pyrrole, we studied the same reaction of $\mathrm{N}$-alkyl derivatives of pyrrolidine, which are readily available from the reaction of dichloro- or dibromobutanes and corresponding amines. $N$-Methyl-, $N$-ethyl, $N$-isopropyl- and $N$ tertbutyl-pyrrolidines all gave the corresponding $N$-alkyl dichloropentathiepinopyrroles 22 as the main product in low to moderate yield $(16-31 \%) \cdot{ }^{20}$ Additionally $N$-methylpyrrolidine gave a small amount $(5 \%)$ of unchlorinated compound 23a with the pentathiepin ring fused across the 2,3-pyrrole bond, $N$-ethylpyrrolidine - monochlorinated product $\mathbf{2 4}$ and $N$-isopropylpyrrolidine bispentathiepin 25, which is the first and only known so far bispentathiepin.

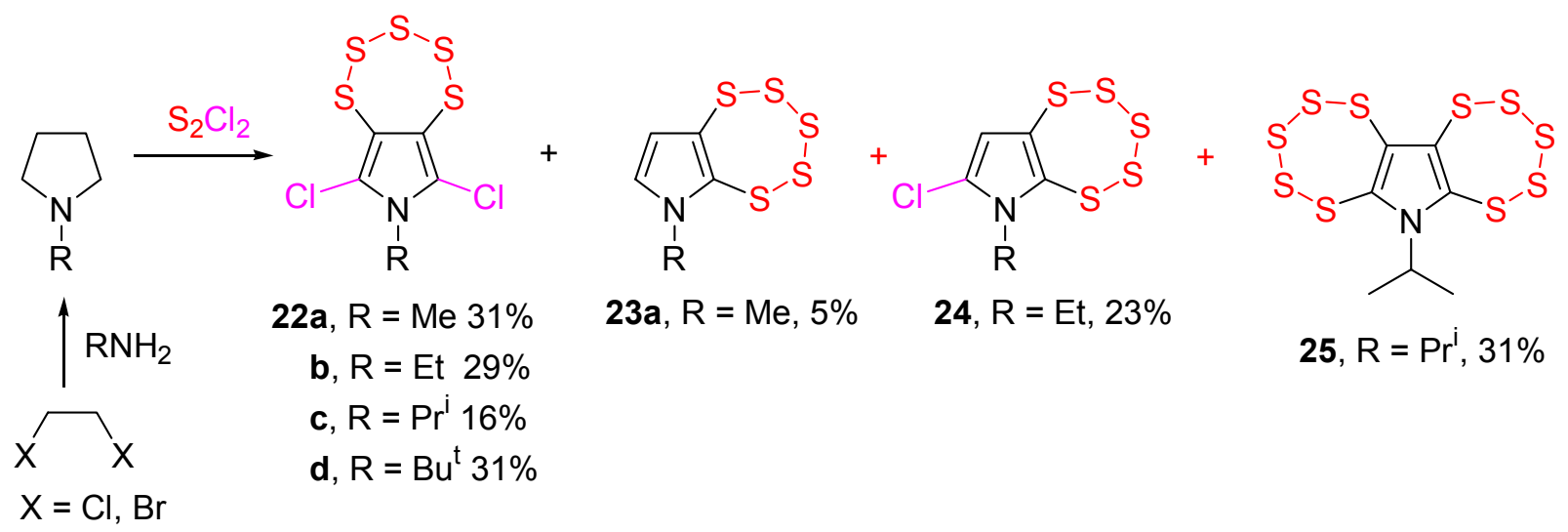

Scheme 14. Reaction of $N$-alkylpyrrolidines with $\mathrm{S}_{2} \mathrm{Cl}_{2}$.

Sulfur monochloride reacted in these transformations simultaneously as sulfurating (formation of pentathiepin ring) and chlorinating (chlorination of pyrrole ring) agent. Obviously complex 2 prepared from sulfur monochloride and two equivalents of DABCO should exhibit mainly sulfurating rather than chlorinating ability and we used it in the reaction with nucleophilic heterocycles. Indeed, the reaction of all $\mathrm{N}$-alkylpyrrolidines with complex 2 gave selectively the $\mathrm{N}$-alkylpentathiepinopyrroles $\mathbf{2 3}$ in moderate yields; no chlorinated products were detected in any of these reactions. ${ }^{7}$ The same products were obtained from $N$-alkylpyrroles, but in that case it is necessary to use a lower amount of complex $\mathbf{2}$ to get selective process: the yields of pentathiepins $\mathbf{2 3}$ were compatible with those obtained from pyrrolidines. $N$-Isopropylpyrrole gave selectively bispentathiepin 25. 


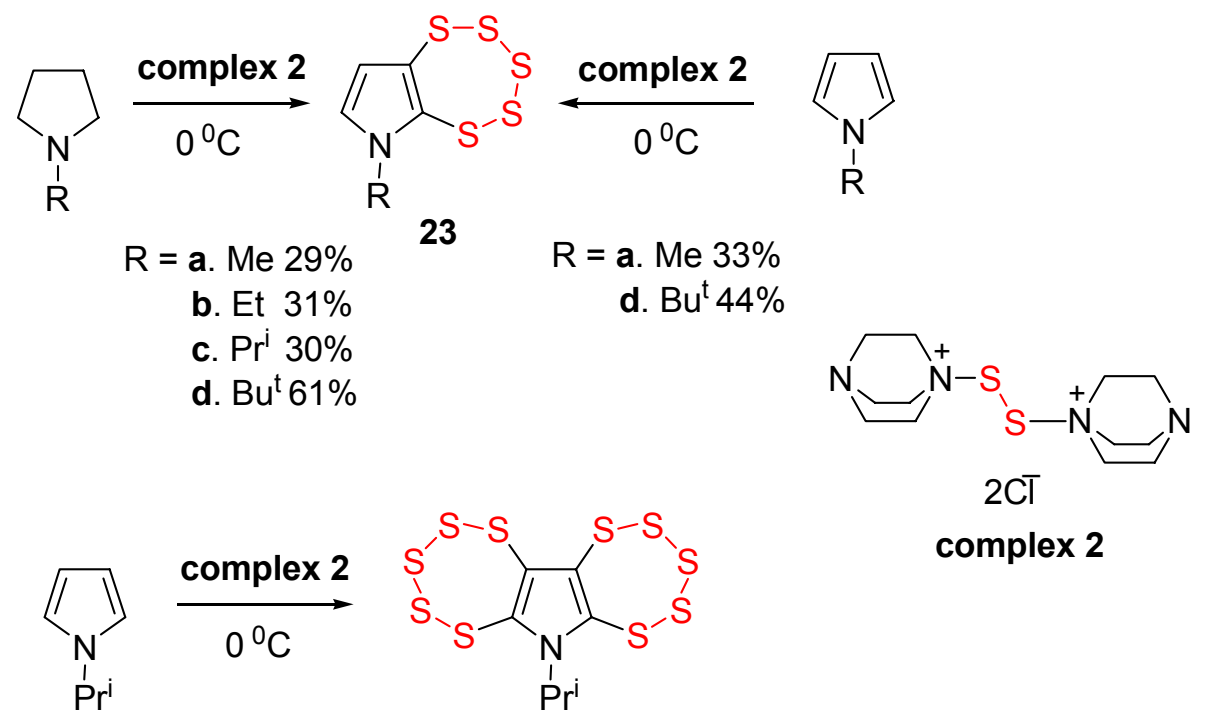

$25,33 \%$

Scheme 15. Selective synthesis of pentathiepinopyrroles 23 and 25.

We tried to spread these reactions to other heterocycles. $N$-Alkylindoles reacted, as pyrroles, to give pentathiepins 26 in moderate yields. Tetrahydrothiophene also afforded corresponding pentathiepin 27 in rather good yield (Scheme 16). Unfortunately, more aromatic heterocycles such as thiophene, benzothiophene and furan did not react with complex 2.
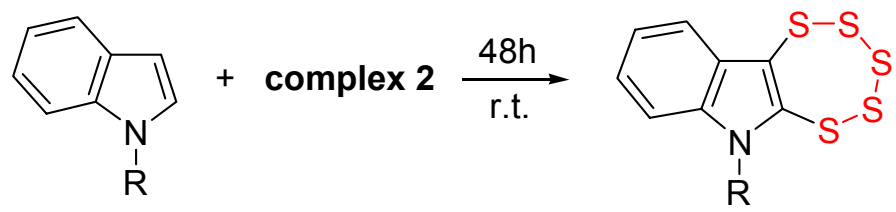

26, $R=$ a, Me $51 \%$
b. Et $62 \%$

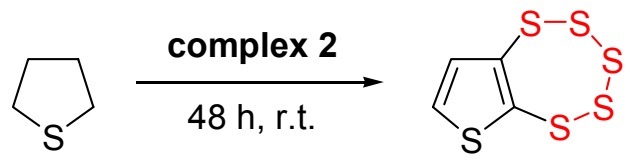

$27,44 \%$

Scheme 16. Reaction of other heterocycles with complex 2.

Consider possible mechanism for the formation of pentathiepins from $N$-methylpyrrolidine as an example (Scheme 17). The key steps are aromatization of the saturated heterocycle and the formation of a $\beta$-annulated pentathiepin ring. $N$-Methylpyrrolidine is proposed to be the first oxidized with complex $\mathbf{2}$ into immonium salt $\mathbf{2 8}$, which then is transformed into enamine 29 
under the action of a base. When repeated, this process yields $N$-methylpyrrole. This is followed by electrophilic addition of complex 2 to position 2 of the heterocycle, giving rise to cation $\mathbf{3 0}$. Then, the chain $-\mathrm{S}_{2} \mathrm{X}$ can be extended, through addition of complex $\mathbf{2}$ and the loss of $\mathrm{SXCl}$, to the chain $-\mathrm{S}_{(\mathrm{n})} \mathrm{X}$ and, finally, cyclize to the thermodynamically stable pentathiepin ring in compound 23a.
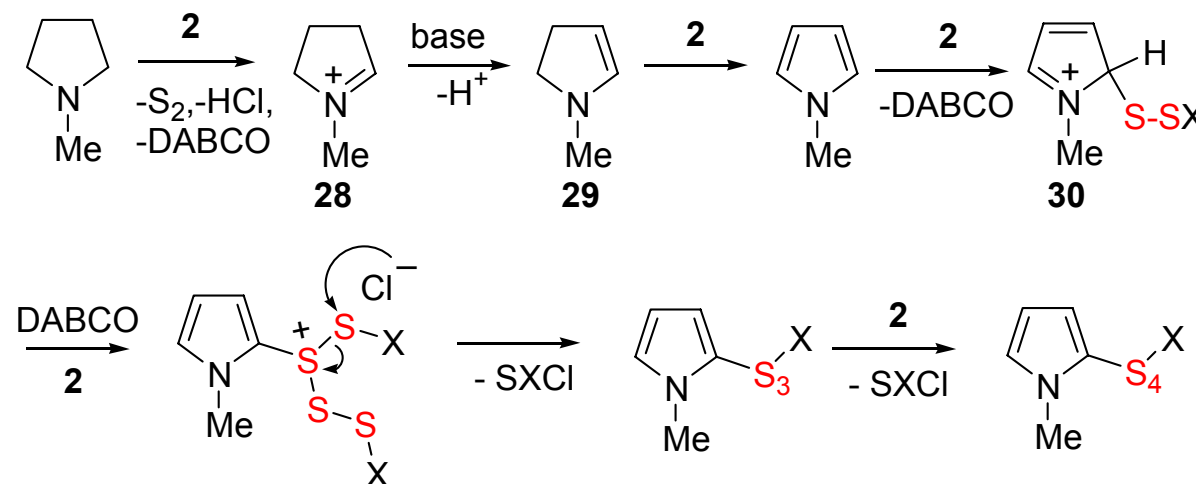

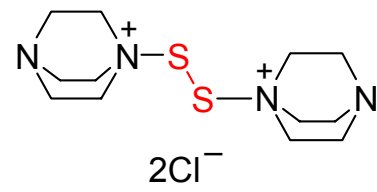

complex 2

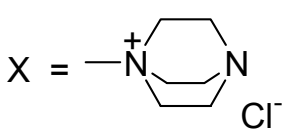

Scheme 17. A plausible mechanism of the $N$-methylpentathiepinopyrrole 23a formation.

If the pyrrole $\alpha$-positions are substituted, the pentathiepin ring is fused across the 3,4-pyrrole bond to give pentathiepinopyrroles 31 in moderate yield. We have further investigated the synthesis of pentathiepinopyrroles from 2,5-dimethylpyrroles, which are readily available from acetonylacetone and amine. It turned out that the best yields were obtained if we used as a reagent complex 1 at low temperature $\left(0{ }^{\circ} \mathrm{C}\right) .{ }^{21}$ The pentathiepins 31 were isolated in moderate yields. Surprisingly isolated and purified pentathiepins 31 were found to react further with complex 1 quite rapidly at a slightly higher temperature $\left(20^{\circ} \mathrm{C}\right)$ to give the bis(dithiolo)pyrroles 32 in high yields (Scheme 18).

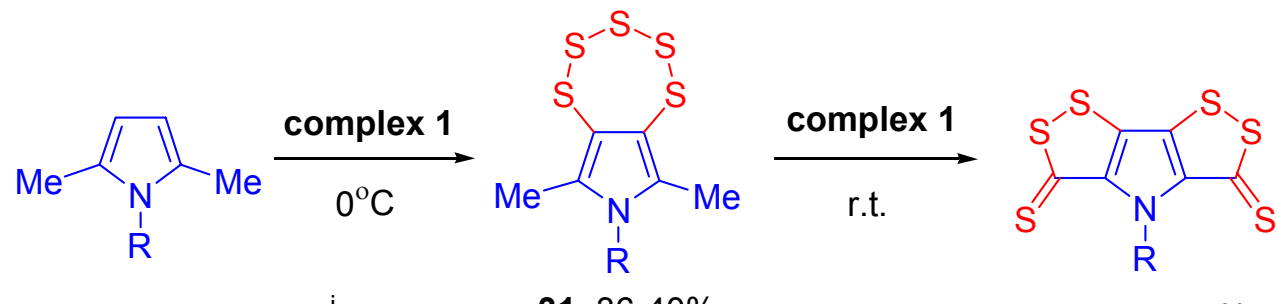

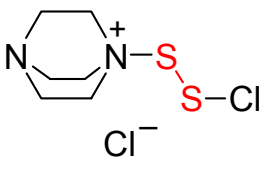

complex 1

Scheme 18. An unusual route from 2,5-dimethylpyrroles to bis(dithiolo)pyrroles 32. 
Although pentathiepin rings and methyl groups are normally unreactive towards $\mathrm{S}_{2} \mathrm{Cl}_{2}$ $\mathrm{DABCO}$ at room temperature, the pyrroles $\mathbf{3 1}$ react in an extensive cascade sequence. Presumably the electron releasing pyrrole nitrogen activates $\mathbf{3 1}$ to attack by the electrophilic reagent, either at the pentathiepin ring or at a methyl group. This reaction cascade provides a new and unusual route from 2,5-dimethylpyrroles to bis(dithiolo)pyrroles 32, some of which have marked activity against various cancer lines. The procedure is better yielding and very much milder than the classical, high temperature $\left(\mathrm{ca} .200^{\circ} \mathrm{C}\right)$ reactions between sulfur and a variety of three-carbon components. ${ }^{22}$

\section{Synthesis of Other Sulfur Containing Heterocycles}

In a search of a new materials, we have examined the reactions of $\mathrm{S}_{2} \mathrm{Cl}_{2}$ in the presence of a chlorinating agent (NCS) and a base (Hünig's base or DABCO), mostly in THF, with 1cyclopentenylacetic acid, 3-indenylacetic acid, its nitrile and 1-(dicyanomethylene) indane. ${ }^{23} \mathrm{We}$ have found that all these compounds gave sometimes completely different types of heterocyclic and polychlorinated derivatives. The cyclopentenylacetic acid yielded purple crystals of the trichlorocyclopenta-1,2-dithiole ester 33, the product of heterocyclic ring formation, chlorination and dehydrochlorination and, unexpectedly, conversion of the acid in THF into its 4-chlorobutyl ester (Scheme 19).

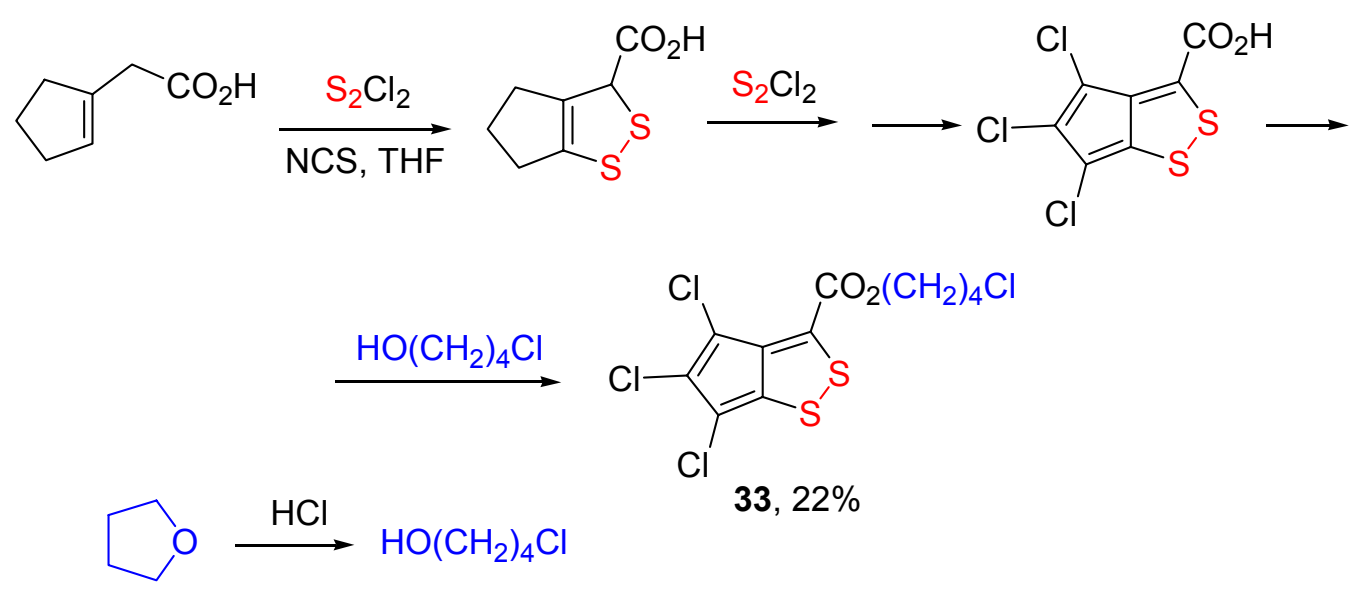

Scheme 19. Reaction of cyclopentenylacetic acid with $\mathrm{S}_{2} \mathrm{Cl}_{2}$.

3-Indenylacetic acid was treated with $\mathrm{S}_{2} \mathrm{Cl}_{2}$, Hünig's base and NCS in various solvents under a variety of conditions and chromatography gave three crystalline products (Scheme 20). One of these did not contain sulfur and is shown by their analytical and spectral properties to be polychlorinated methyleneindene $\mathbf{3 4}$, obtained in up to $32 \%$ yield. This clearly arose by extensive chlorination and dehydrochlorination of indenylacetic acid, a standard feature of many $\mathrm{S}_{2} \mathrm{Cl}_{2}$ reactions, accompanied 
by chlorodecarboxylation. The other two products $\mathbf{3 5}$ and $\mathbf{3 6}$ had incorporated sulfur to form novel and unexpected heterocyclic systems.

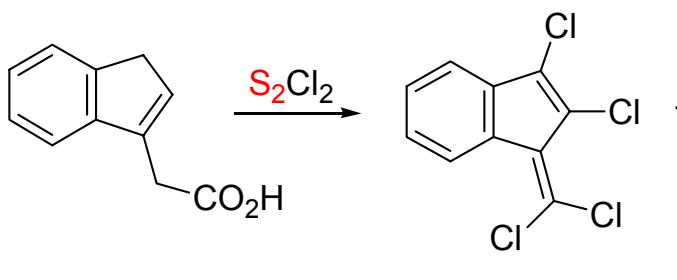

$34,32 \%$<smiles>O=c1ssc2c1-c1ccccc1C2(Cl)Cl</smiles>

$35,15 \%$<smiles>O=C1SC2=C(Cl)c3ccccc3C2=C1Cl</smiles>

$36,18 \%$

Scheme 20. Reaction of 3-indenylacetic acid with $\mathrm{S}_{2} \mathrm{Cl}_{2}$.

Whilst the conversion of indenylacetic acid into the major indene product 34 was not predicted, a pathway for two cyclic products can be readily envisaged. The formation of the heterocycle 35 based on the demonstrated propensity for $\mathrm{S}_{2} \mathrm{Cl}_{2}, \mathrm{NCS}$ and Hünig's base to form 1,2-dithiole rings with activated allylic systems, followed by extensive chlorination dehydrochlorination to give fully unsaturated and chlorinated products. This assumes that the acid group is lost by decarboxylation, possibly to give a 3-chloro-1,2-dithiolium chloride which then reacts with some external oxygen nucleophile, as we have proposed in related reactions. Formation of the fused thiophenone 36 is, we believe, rare in $\mathrm{S}_{2} \mathrm{Cl}_{2}$ reactions. Since $\mathrm{S}_{2} \mathrm{Cl}_{2}$ has been shown to convert carboxylic acids into acid chlorides, it is possible that some intermediate in this transformation could be diverted by cyclization to 1,2-dithiine derivative and ultimately to form thiophene with sulfur extrusion.

The dicyanomethylene indane gives the dichloroindene $\mathbf{3 7}$ and the red, thermochromic indeno-1,2-thiazine $38 .{ }^{24}$ A reasonable pathway for the conversion of dicyanide into thiazine is given in Scheme 21. Addition of $\mathrm{S}_{2} \mathrm{Cl}_{2}$ to a nitrile bond followed by cyclization onto an activated allylic position would give the dithiazepine 39. Standard chlorination-dehydrochlorination followed by sulfur extrusion would then give the planar and formally aromatic product $\mathbf{3 8}$ isolated. 


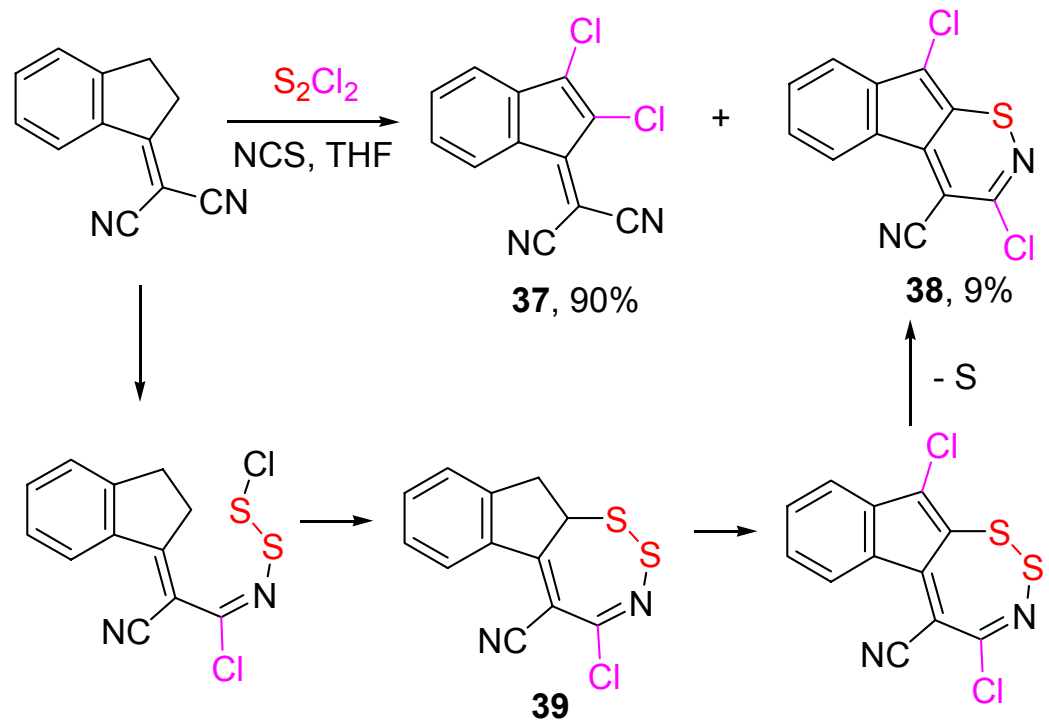

Scheme 21. Reaction of dicyanomethylene indane with $\mathrm{S}_{2} \mathrm{Cl}_{2}$.

Many of these products interestingly showed birefringence on heating in a hot stage polarising microscope, indicating liquid crystalline behaviour and constitute a new classes of discotic liquid crystals in which the molecular order of the mesophases is supported by intermolecular interactions. $^{25}$

We have shown (see Section 2) that $N$-isopropyl groups can be converted by $\mathrm{S}_{2} \mathrm{Cl}_{2}$ into $N$ (1,2-dithiole-3-thiones). Nitrogen heterocycles containing methyl and $\mathrm{C}-\mathrm{H}$ groups in orthopositions, such as easily available and even commercial 2-methylindoles, are structurally similar to the isopropyl group and may be considered as potential intermediates in the synthesis of dithioloindole thiones. Fused 1,2-dithole-3-thiones have never been generated from methyl heterocycles. We have found that the reaction of 1,2-dimethylindole 40a with a fivefold excess of complex 1 in chloroform for $48 \mathrm{~h}$ at room temperature led after treatment with $\mathrm{Et}_{3} \mathrm{~N}$ to dithiole thione 41a in moderate yield $(34 \%) .{ }^{26}$ No wonder that complex 2 , which is a more selective sulfurating agent, reacted with 40a in the same conditions more cleanly to afford thione 41a in high yield (94\%) (Scheme 22). Further on, we extended this reaction to other $N$-substituted 2methylindoles 40. Fused dithioloindoles 41 were obtained in a reaction with $N$-alkyl- and $N$ benzyl-2-methylindoles $\mathbf{4 0}$ in moderate to high yields. $N$-Acetyl- and $N$-benzoyl-2-methylindoles did not react with $\mathrm{S}_{2} \mathrm{Cl}_{2}$ and their complexes 1 and $\mathbf{2}$ even under reaction mixtures refluxing for 5 $\mathrm{h}$ in chloroform; starting indoles $\mathbf{4 0}$ were isolated from the reaction mixtures. Apparently electron-withdrawing substituents at nitrogen in indole (acetyl or benzoyl groups) suppress the reaction with $\mathrm{S}_{2} \mathrm{Cl}_{2}$ even under more vigorous conditions. 


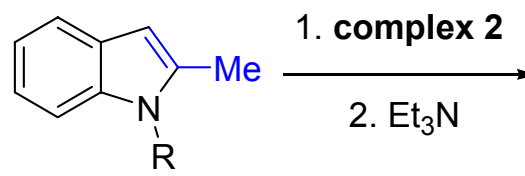

40

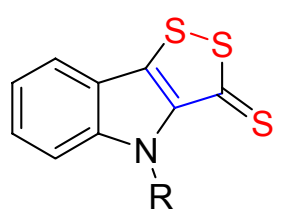

41

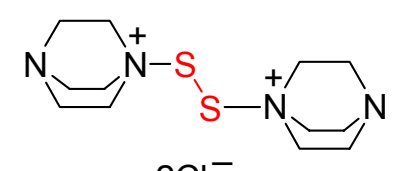

$2 \mathrm{Cl}^{-}$

complex 2

$$
\begin{aligned}
& \text { a } \mathrm{R}=\mathrm{Me}, 94 \% \\
& \text { b } \mathrm{R}=\mathrm{Et}, 56 \% \\
& \text { c } \mathrm{R}=\mathrm{Pr}^{\mathrm{i}}, 66 \% \\
& \text { d } \mathrm{R}=\mathrm{Bn}, 70 \%
\end{aligned}
$$

Scheme 22. Synthesis of $N$-substituted [1,2]dithiolo[4,3-b]indole-3(4H)-thiones 41.

1,2,3-Dithiazoles have attracted much attention among five-membered sulfur-nitrogen heterocycles because of their interesting physical and biological properties and versatile chemistry. ${ }^{27}$ The abundance of monocyclic dithiazole chemistry has been derived from 4,5dichloro-1,2,3-dithiazolium chloride - Appel salt - which is readily prepared from chloroacetonitrile and sulfur monochloride. Surprisingly, other than Appel salt 4-substituted 1,2,3-dithiazolium chlorides are hardly available. We have found ${ }^{28}$ that 5-one- 42, 5-thione- 43 and 5-phenylimino-1,2,3-dithiazoles 44 could be selectively obtained in one pot reaction of various ethanone oximes with sulfur monochloride and pyridine in acetonitrile followed by treatment by corresponding nucleophiles (formic acid, thioacetamide and aniline) in high to moderate yields (Scheme 23). Apparently the key intermediates in the formation of 1,2,3dithiazoles were 1,2,3-dithiazolium chlorides $\mathbf{4 5}$ which were found to be unstable and converted to keto, thio and arylimino derivatives by the reaction with corresponding nucleophiles in situ. The yield of dithiazoles $\mathbf{4 2 - 4 4}$ was found to be sensitive to the reaction conditions: the reaction time and temperature, the nature of solvent and a base used. The best protocol for the preparation of dithiazoles 42-44 included reaction of ethanone oxime with $\mathrm{S}_{2} \mathrm{Cl}_{2}$ (2.1 equiv) and pyridine (2.8 equiv) in acetonitrile at $0^{\circ} \mathrm{C}$ for 15 min following treatment with an excess of corresponding nucleophile. 


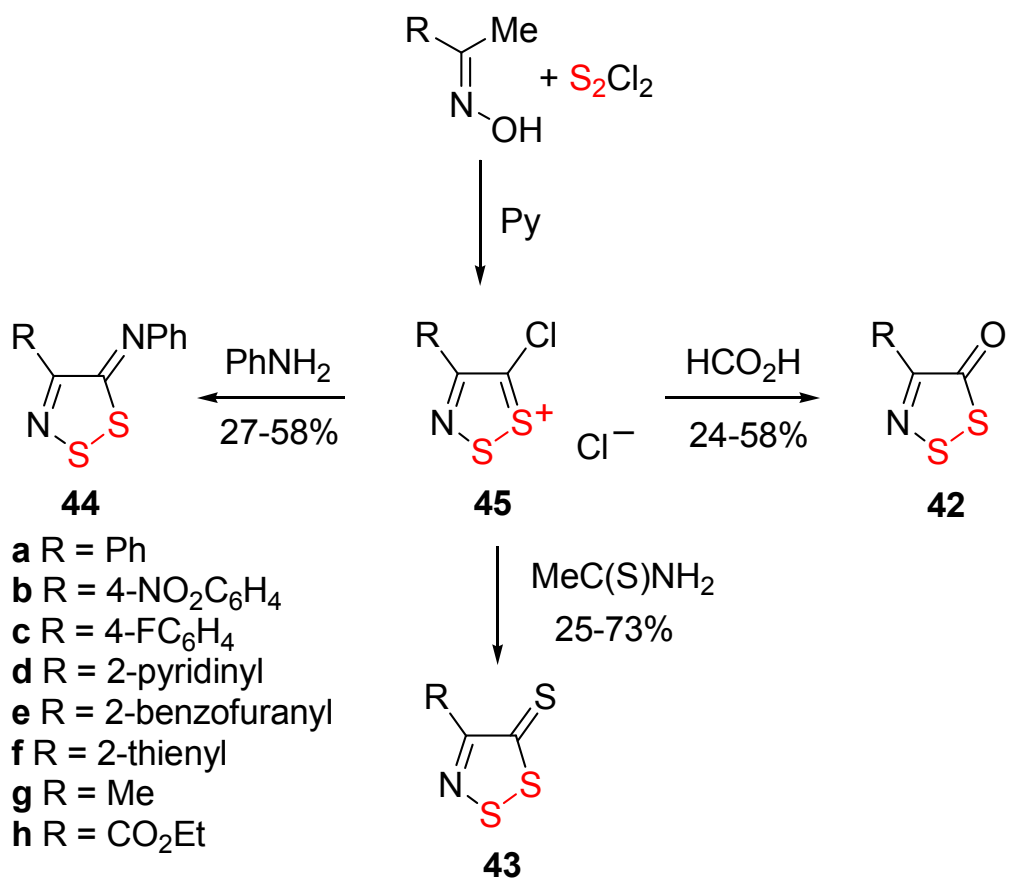

Scheme 23. Synthesis of 1,2,3-dithiazoles.

\section{Conclusions}

The reactions described show that the presented methodology constitutes a fast and convenient route to sulfur heterocyclic systems that in many cases are not easy, or impossible to obtain by conventional ways. A wide scope of new polysulfur-nitrogen heterocycles, their interesting characteristics and rapid synthetic methods from readily available materials make this strategy very promising. The limit of this chemistry is difficult to foresee.

\section{Acknowledgments}

We gratefully acknowledge financial support from the Royal Society, the Russian Foundation for Basic Research (grants No. 05-03-32032, 08-03-00003) and the Royal Society of Chemistry (Research Fund Grants to O.A.R.).

\section{References}

1. Rakitin, O. A.; Konstantinova, L. S. Adv. Heterocycl. Chem., 2008, 96, 175.

2. Ballester, M.; Molinet, C.; Castander, J. J. Am. Chem. Soc. 1960, 82, 4294. 
3. Garcia, R.; Riera, J.; Carilla, J.; Julia, L.; Molins, E.; Miravitlles, C. J. Org. Chem. 1992, 57, 5712.

4. Torroba, T. J. Prakt. Chem. 1999, 341, 99.

5. García-Valverde, M.; Torroba, T. Eur. J. Org. Chem. 2006, 849.

6. Kudo, Y.; Hamada, S. Bull. Chem. Soc. Jpn. 1983, 56, 2627.

7. Konstantinova, L. S.; Rakitin, O. A.; Rees C. W.; Amelichev, S. A. Mendeleev Commun. 2004, 91.

8. Marcos, C. F.; Polo, C.; Rakitin, O. A.; Rees C. W.; Torroba, T. Angew. Chem., Int. Ed. Engl. 1997, 36, 281.

9. Rees, C. W.; White, A. J. P.; Williams, D. J.; Rakitin, O. A.; Marcos, C. F.; Polo C.; Torroba, T. J. Org. Chem. 1998, 63, 2189.

10. Barriga, S.; Konstantinova, L. S.; Marcos, C. F.; Rakitin, O. A.; Rees, C. W.; Torroba, T.; White A. J. P.; Williams, D. J. J. Chem. Soc., Perkin Trans. 1 1999, 2237.

11. Konstantinova, L. S.; Rakitin O. A.; Rees, C. W. Mendeleev Commun. 2001, 165.

12. Amelichev, S. A.; Barriga, S.; Konstantinova, L. S.; Markova, T. B.; Rakitin, O. A.; Rees, C. W.; Torroba, T. J. Chem. Soc., Perkin Trans. 1 2001, 2409.

13. Konstantinova, L. S.; Rakitin, O. A.; Rees, C. W. Mendeleev Commun. 2001, 167.

14. Konstantinova, L. S.; Obruchnikova, N. V.; Rakitin, O. A.; Rees, C. W.; Torroba, T. J. Chem. Soc., Perkin Trans. 1 2000, 3421.

15. Rees, C. W.; White, A. J. P.; Williams, D. J.; Rakitin, O. A.; Konstantinova, L. S.; Marcos, C. F.; Torroba, T. J. Org. Chem. 1999, 64, 5010.

16. Konstantinova, L. S.; Berezin, A. A.; Lysov, K. A.; Rakitin, O. A. Russ. Chem. Bull. 2006, 143.

17. Konstantinova, L. S.; Berezin, A. A.; Lysov, K. A.; Rakitin, O. A. Tetrahedron Lett. 2007, $48,5851$.

18. Konstantinova, L. S.; Rakitin, O. A.; Rees, C. W.; Souvorova, L. I.; Golovanov, D. G.; Lyssenko, K. A. Org. Lett. 2003, 5, 1939.

19. Konstantinova, L. S.; Rakitin, O. A.; Rees, C. W. Chem. Commun., 2002, 1204.

20. Amelichev, S. A.; Konstantinova, L. S.; Lyssenko, K. A.; Rakitin, O. A.; Rees, C. W. Org. Biomol. Chem. 2005, 3, 3496.

21. Amelichev, S. A.; Aysin, R. R.; Konstantinova, L. S.; Obruchnikova, N. V.; Rakitin, O. A.; Rees, C. W. Org. Lett. 2005, 7, 5725.

22. Pedersen, C. Th. Sulfur Rep. 1995, 16, 173.

23. Konstantinova, L. S.; Rakitin, O. A.; Rees, C. W.; Souvorova, L. I.; Torroba, T.; White, A. J. P.; Williams, D. J. Chem. Commun. 1999, 73.

24. Konstantinova, L. S.; Rakitin, O. A.; Rees, C. W.; Souvorova, L. I.; Torroba, T. J. Chem. Soc., Perkin Trans. 1 1999, 1023.

25. Barbera, J.; Rakitin, O. A.; Ros, M. B.; Torroba T. Angew. Chem., Int. Ed. 1998, 37, 296.

26. Konstantinova, L. S.; Lysov, K. A.; Amelichev, S. A.; Obruchnikova, N. V.; Rakitin O. A. Tetrahedron 2009, 65, 2178. 
27. Konstantinova, L. S.; Rakitin, O. A. Russ. Chem. Rev. 2008, 77, 521.

28. Konstantinova, L. S.; Bol'shakov, O. I.; Obruchnikova, N. V.; Laborie, H.; Tonga, A.; Sopéna, V.; Lanneluc, I.; Picot, L.; Sablé, S.; Thiéry, V.; Rakitin, O. A. Bioorg. Med. Chem. Lett. 2009, 19, 136.

\section{Author's biography}

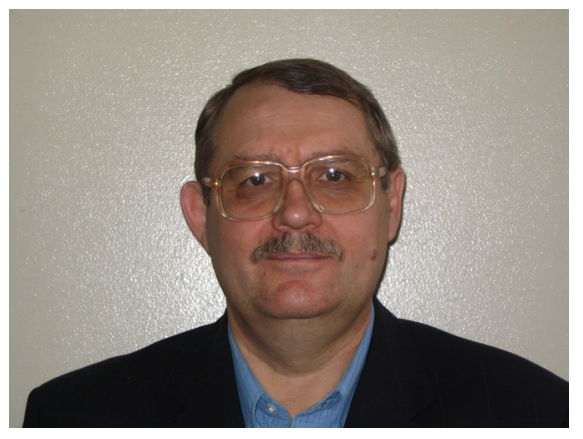

Oleg Rakitin was born in Moscow. He graduated at M. V. Lomonosov Moscow State University in 1974. He was a Junior Researcher, Senior Researcher, and since 1995 Head of the Laboratory at N. D. Zelinsky Institute of Organic Chemistry. He received his Ph.D. in 1980 and his Doctor of Science in 1992. He has spent a few months in the laboratory of Professor C. W. Rees at Imperial College (London, UK) being awarded a Royal Society Kapitza Fellowship, a Royal Society of Chemistry three Journals Grant for International Authors and four Royal Society Joint Projects. He has published over 100 scientific papers including 15 reviews and book chapters. His scientific interests include the synthesis and chemistry of nitrogen and sulfur heterocyclic compounds, particularly polysulfur heterocycles. 